\title{
Japanische Gärten in Kyoto
}

\author{
Heidrun Janka \& Andreas Hamacher
}

\begin{abstract}
The city of Kyoto was emperial residence for more than 1000 years and soon became the most prosperous cultural centre of Japan. Garden and landscape design, beside other performing arts such as painting, sculpture, calligraphy and architecture evolved to diverse styles during a long creative period. Each of the diverse garden designs represents a specific cultural era in Japanese history and is strongly connected to buddhism, especially Zen-buddhism. Numerous Japanese gardens can be visited in the vicinity of the large temple complexes, shrines and monasteries at Kyoto, including various pond and strolling gardens, dry and rock gardens.
\end{abstract}

\section{Zusammenfassung}

Die Stadt Kyoto war über 1000 Jahre lang Sitz des kaiserlichen Hofes von Japan und entwickelte sich dadurch frühzeitig zum bedeutendsten Kulturzentrum des Landes. Neben der Architektur, Malerei, Kalligraphie und Bildhauerei hat die Gartenkunst in Kyoto eine besondere schöpferische Vielfalt erfahren. In der mehr als tausendjährigen Gartengeschichte Japans haben sich unterschiedliche Gartenstile entwickelt, die Ausdruck bestimmter zeitlicher Epochen sind. Einen starken Einfluss auf die Gartenkunst in Japan hatten verschiedene Ausprägungen des Buddhismus, insbesondere der Zen-Buddhismus. In Kyoto können im Umkreis von Tempeln, Klöstern und Schreinen eine große Vielfalt verschiedener japanischer Gärten besichtigt werden, darunter Teich-, Wandel-, Stein-, Tee- und Hofgärten.

\section{Einführung}

Die Stadt Kyoto liegt im mittleren Teil der japanischen Hauptinsel Honshu südwestlich des Biwa-Sees in einem von Hügeln umgebenen Becken zwischen den Flüssen Katsura und Kamo (Abb. 1). Bedingt durch die Tal-Lage staut sich in Kyoto während der warmen Sommermonate im Juli und August feuchtwarme Luft. Das durchschnittliche Temperatur-Maximum liegt in dieser Zeit bei $27,7^{\circ} \mathrm{C}$. Die Hauptregenzeit ist im Juni. Während der Wintermonate ist es dagegen trocken und kühl, aber frostfrei. Im Jahresdurchschnitt liegen die Niederschlagswerte in Kyoto bei $1581 \mathrm{~mm}$ und die Temperatur bei $15,3{ }^{\circ} \mathrm{C}$.

Kyoto ist heute die siebtgrößte Stadt Japans, Hauptstadt der gleichnamigen Präfektur und bildungspolitisches Zentrum in West-Japan (GIESEN et al. 2007). Die Umgebung von Kyoto ist eines der modernsten und größten Industriezentren des Landes. Kyoto bietet viele Kontraste. Einerseits gibt es dort sehr moderne japanische Architektur und Lebenskultur, zum anderen trifft man auf Zeugen des reichen kulturellen Erbes aus verschiedenen Epochen der Geschichte Japans. Die zahlreichen Tempelanlagen, Schreine und Gärten sind größtenteils erhalten geblieben und an vielen Orten in der Stadt anzutreffen.
Kyoto war von 794 bis 1868 Sitz des kaiserlichen Hofes von Japan. Dadurch entwickelte sich die Stadt früh zum bedeutendsten Kulturzentrum des Landes, in dem Architektur, Gartenkunst, Malerei, Bildhauerei und viele andere Künste zu höchster Blüte gelangten. Religiöse Einflüsse kamen bereits im 6. Jh. von China nach Japan. Der Buddhismus hatte maßgeblichen Einfluss auf die Kunst und Kultur Japans, was sich in Kyoto in der großen Anzahl (insgesamt 1650) erhalten gebliebener Tempel und in den darum angelegten Gärten zeigt. In der Stadt existieren zudem rund 200 Shinto-Schreine, die Zeugen eines noch viel älteren, in Japan begründeten mythologischen Ahnenkults (Shintoismus) sind (GIEsEn et al. 2007). Der großen Vielfalt außergewöhnlich schöner Gärten ist zu verdanken, dass Kyoto als Zentrum der japanischen Gartenkunst gilt. Die Stadt ist für Garten- und Kunstinteressierte die Haupt-Attraktion einer Japan-Reise. Über tausend Jahre Gartengeschichte aus verschiedenen Epochen der Geschichte Japans haben teilweise sehr unterschiedliche Stile hervorgebracht, die man heute in den zahlreichen Teich- und Bachgärten, Wandelgärten, Stein-, Tee- und Hofgärten sehen kann. 

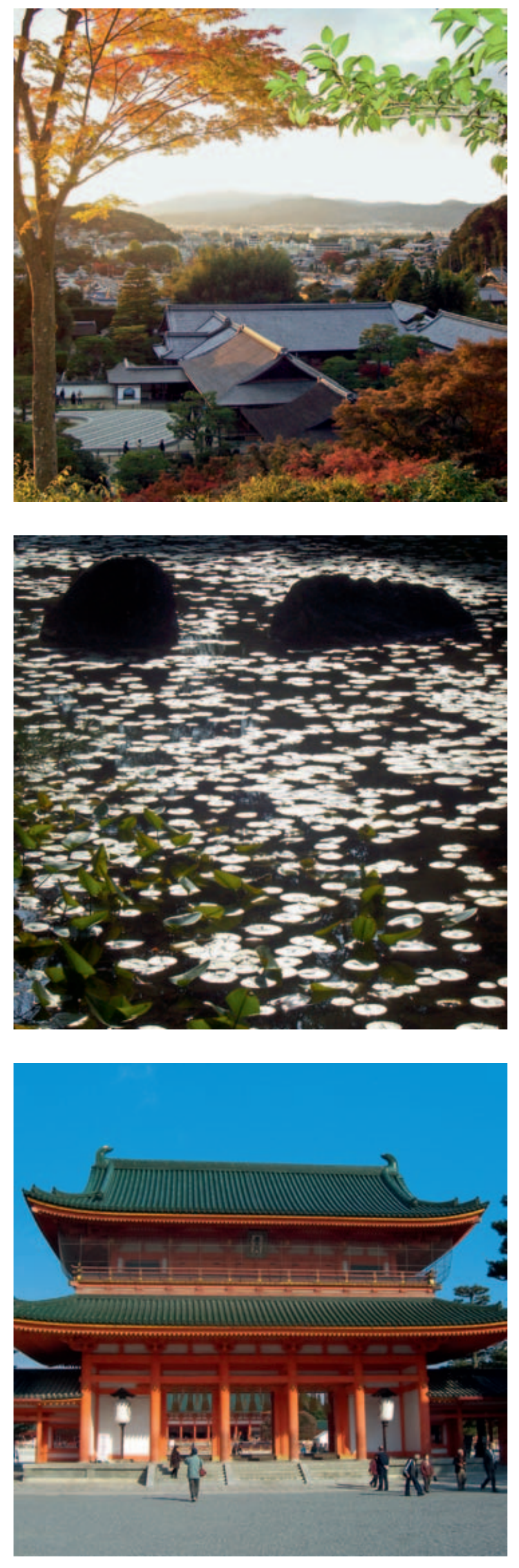

\section{Gärten der Heian-Zeit (794-1185)}

Mit der Verlegung der Hauptstadt und der Kaiserresidenz von Nara nach Heian-kyo (Kyoto) im Jahre 794 begann in Japan eine Zeit des Wohlstands und der kulturellen Blüte. Die neue Stadt Heian-kyo wurde nach Vorbild der chinesischen Stadt Xian mit einem schachbrettartigen Grundriss angelegt, was sich heute noch in einer quadratischen Form der Gartenanlagen widerspiegelt (CHEsshire 2007). Kunsthandwerk und Gartenarchitektur wurden in der Heian-Zeit stark gefördert; man legte viel Wert auf Details, Rituale und jahreszeitliche Aspekte. Diese Zeit galt daher als romantischste Ära der japanischen Kulturgeschichte. Ein wesentlicher Bestandteil von Gärten der Heian-Zeit waren Teiche und Inseln, die in Bezug zur chinesischen Mythologie standen (Chesshire 2007). Der Legende zufolge lebten die Unsterblichen auf sagenhaften Inseln im Meer, die auf dem Rücken von Schildkröten getragen wurden. Die Unsterblichen wurden durch Kraniche auf die Inseln getragen. Die mystischen Inseln wurden in den Gärten in Form von besonders angeordneten Felsgruppen in Seen dargestellt (Abb. 2). Man erhoffte sich, durch deren Nachbildung die Unsterblichen anzulocken, um das Elixier der ewigen Jugend mit ihnen zu teilen. Außerdem stellte man sich so das paradiesische Land der Reinheit des Buddha Amida vor. Im Zuge des immer mehr an Einfluss gewinnenden Buddhismus wandelten sich die mystischen Felsinseln ab dem 6. Jh. zum Sinnbild des heiligen Berges Shumisen. Dieser galt als Mittelpunkt der buddhistischen Mythologie und stellt ein wichtiges Berg-Wasser-Motiv in der japanischen Kunstform dar. Hierdurch verschmolzen bud-

Abb. 1 (oben): Blick von der Anhöhe des Kodai-ji-Gartens auf die Stadt Kyoto.

Abb. 2 (Mitte): Felsen im Teich des Ginkaku-ji Gartens in Kyoto symbolisieren die mystischen Inseln der Unsterblichen.

Abb. 3 (unten): Der Heian-Schrein wurde im Jahre 1895 zum Gedenken an den elfhundertsten Jahrestag der Stadtgründung Kyotos errichtet und ist dem letzten dort residierenden Kaiser KomeI gewidmet. Die Anlage stellt eine verkleinerte Kopie des ersten Kaiserpalastes von 794 dar. 
dhistische mit den zuvor dominierenden shintoistischen Elementen (CHEsshire 2007).

Im 11. Jh. entstand das Sakuteiki, die weltweit vermutlich erste schriftliche Abhandlung über Gartengestaltung (Young \& Young 2006). Sie nennt viele Formen von Teichen, Inseln sowie Bächen und gibt Ratschläge, wie Bäume zu pflanzen sind. Für die Suche nach geeigneten Standorten für Felsen oder die Wahl von Bachläufen heißt es, man solle dem „Wunsch“ oder der Forderung eines Steins und des Wassers entsprechen. In Japan herrscht nach wie vor die Auffassung, dass unbelebte Natur (Felsen, Steine etc.) eine Individualität besitzt, die mit Respekt zu behandeln sei. Nur bei Beachtung dieses Grundsatzes erreicht man ein harmonisches Ganzes.

Der Heian-Schrein (Abb. 3) in Kyoto ist eine verkleinerte Nachbildung des ersten Kaiserpalastes von 794 und wurde im Jahre 1895 zum Gedenken des elfhundertsten Jahrestages der Stadtgründung erbaut (Keane 1999, Chesshire 2007). Der heilige Garten Shinen des HeianSchreins in Kyoto ist im Stil von Gärten aus der Heian-Zeit des 10. und 11. Jh. gestaltet. Im Zentrum des romantischen Landschaftsgartens liegt ein großer Boots-See mit Kiefern bewachsenen Inseln (Abb. 4, 5). Die Kiefern (Pinus thunbergii) sind elegant geschnitten und bieten mit ihrer Spiegelung im Wasser einen sehr schönen Anblick. Vor der japanischen Küste vorkommende Kiefern-Inseln dienten bei der Gestaltung als Vorbild. Auf zahlreichen, im Wasser

Abb. 4 (oben): Ein großer Boots-See liegt im Zentrum des heiligen Gartens Shinen am Heian-Schrein in Kyoto. Während der Heian-Zeit wurden auf Boots-Seen prächtige Feste auf bemalten Drachenbooten gefeiert, bei denen musiziert wurde und Gedichte rezitiert wurden.

Abb. 5 (Mitte): Ein wichtiges gestalterisches Element von japanischen Teichgärten sind Kiefern-bewachsene Inseln. Die elegant geschnittenen Bäume (Pinus thunbergii) im heiligen Garten Shinen spiegeln sich sehr schön im Wasser.

Abb. 6 (unten): Yoshino-Kirschbäume (Prunus x yedoensis) säumen das Ufer des Boots-Sees im heiligen Garten Shinen in Kyoto und werden hier durch sehr ausgefeilte Gerüste gestützt. Zur Zeit der Kirschblüte im April bieten die Bäume einen wundervollen Anblick und locken viele Besucher an, die dort das alljährliche Kirschblütenfest feiern.
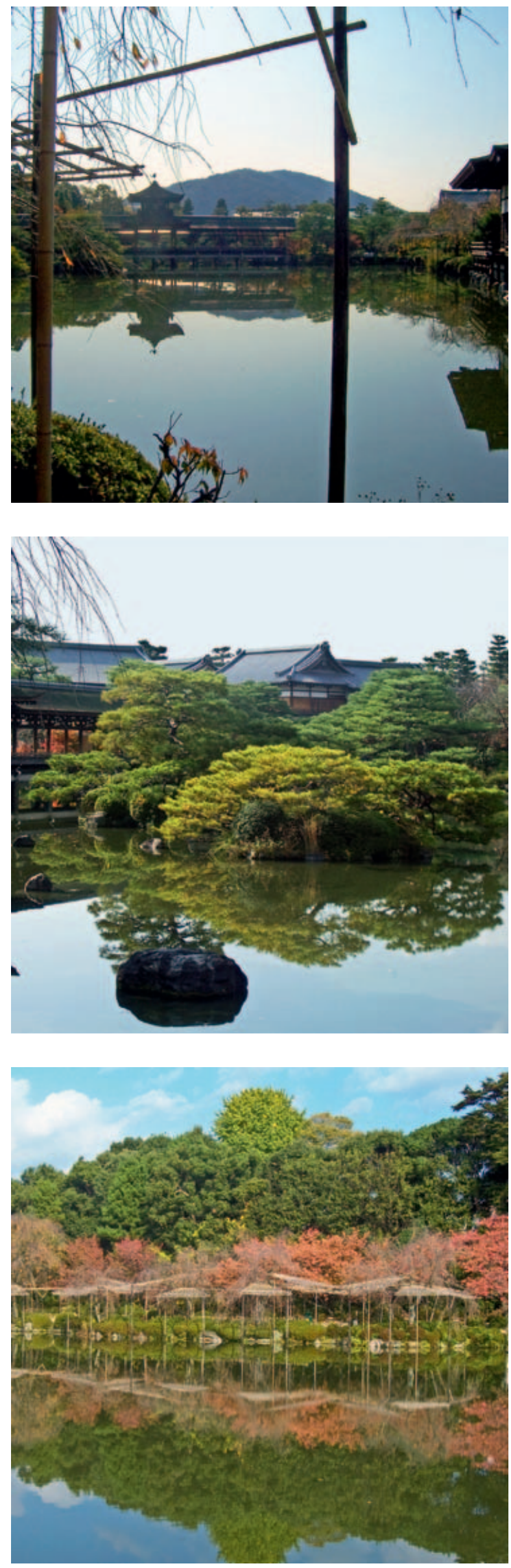

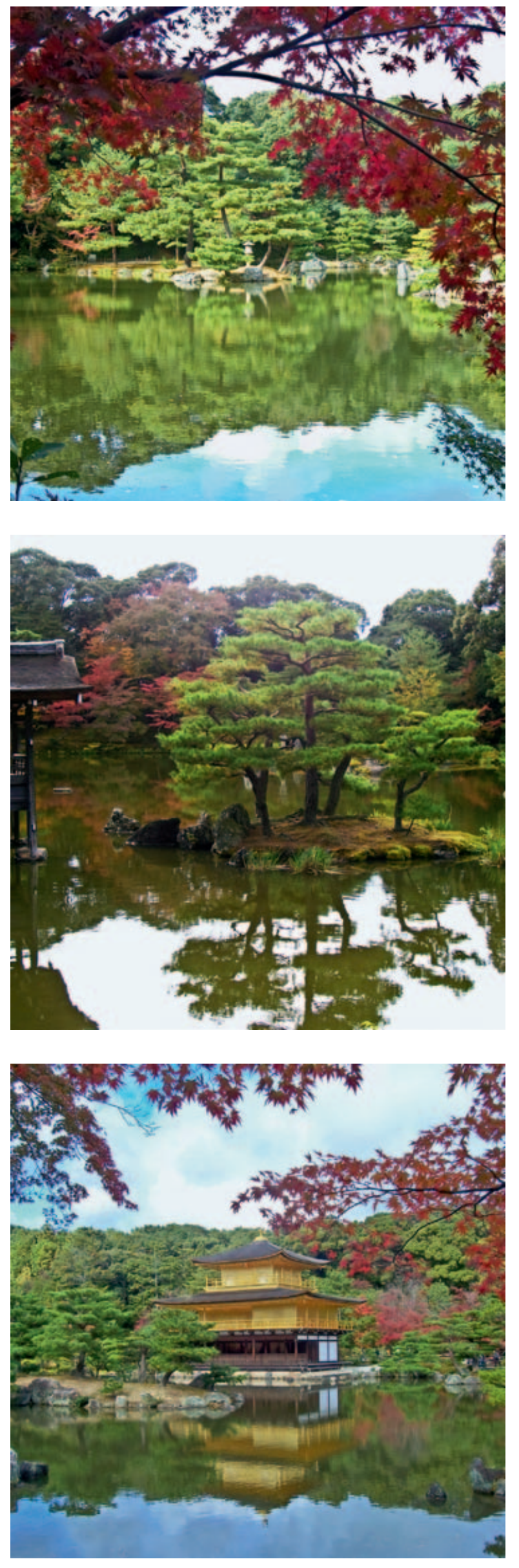

angeordneten Trittsteinen lässt sich der große See durchqueren. Der heilige Garten Shinen ist für seine Yoshino-Kirschbäume (Prunus x yedoensis, Abb. 6) bekannt. Ihre zartrosa Blütenwolken locken zur Zeit des Kirschblüten-Festes (Hanami) im April viele Besucher an.

Während der Heian-Zeit trugen japanische Damen sehr aufwendige Kleider. Es war ihnen dadurch nicht möglich, zwanglos durch den Garten spazierenzugehen. Vielmehr war es für die Damen derzeit üblich, an Bootsausflügen teilzunehmen und den Garten vom Wasser aus zu genießen. Auf Seen wurden prächtige Feste abgehalten, bei denen bemalte Drachenboote mit Musikern in sehr aufwendiger Kostümierung um den See gerudert oder gestakt wurden. Dabei rezitierte man Gedichte, es wurde gesungen und musiziert. Die Natur im Wandel der Jahreszeiten war ein in der Kunst und Literatur wichtiger Aspekt, so wurde beispielsweise die wechselnde Farbe der Ahornblätter oft thematisiert (Abb. 7, Keane 1999). Fächer-Ahorn (Acer palmatum) wurde oft um Boots-Seen herum angepflanzt, um entweder vom Wasser oder von einem Bootshaus bzw. Pavillon aus seine prächtige Laubfärbung zu genießen (Abb. 8). Die wichtigsten Gestaltungselemente von Gärten aus der Heian-Zeit sind große Seen mit baumbewachsenen Inseln, Felsformationen im Wasser, Bootshäuser oder Pavillons und chinesische Brücken; wichtige Pflanzen sind Kiefern (z. B. Pinus thunbergii), Zierkirschen (z. B. Prunus serrulata) und Fächer-Ahorn (Keane 1999, Young \& Young 2006, Chesshire 2007).

Abb. 7 (oben): Laubfärbung der Fächerahorne (Acer palmatum) am Ufer des Kinkaku-ji-Sees in Kyoto. Durch die Spiegelung im Wasser wirkt der Herbstaspekt noch intensiver.

Abb. 8 (Mitte): Bootshaus am Ufer des Kinkaku-ji-Sees in Kyoto mit vorgelagerter Kieferninsel.

Abb. 9 (unten): Der Goldpavillon in Kyoto wurde Ende des 14. Jh. von den ersten Ashikaga-Shogunen zu Beginn der Muromachi-Zeit errichtet. Die oberen Stockwerke des dreistöckigen Tempels sind komplett mit Blattgold überzogen. Seine Schönheit kommt insbesondere durch die Spiegelung im Teich und der geschickten Einbettung in den Landschaftsgarten zur Geltung. 


\section{Gärten der Muromachi-Zeit (1338-1573)}

Während der Regierungszeit der Ashikaga-Shogune entbrannten im ganzen Land bürgerkriegsähnliche Konflikte, die über ein Jahrhundert andauerten. Trotzdem entwickelten sich in dieser unruhigen Zeit Kunst und Kultur sehr beständig, so die Teezeremonie und die Kunst des Blumensteckens (Ikebana). Außerdem wurden neue Stile in der Gartenkunst und -architektur begründet (CHesshire 2007). 1390 entstanden in Kyoto der berühmte Goldpavillon (Kinkakuji, Abb. 9) sowie um 1470 der Silberpavillon (Ginkaku-ji). Beide sind Prunkbauten der Militärherrscher, mit denen Macht und Wohlstand demonstriert wurden. Um die Pavillons herum wurden so genannte Wandelgärten angelegt, die eine stilistische Weiterentwicklung der Gärten aus der Heian-Zeit darstellten (KeAne 1999). Wandelgärten haben sehr viel kleinere Seen als die früheren Boots-Seen und wurden auch nicht mehr für Bootsausflüge genutzt. Um einen zentralen See herum wurden Wandelpfade angelegt, die sich durch Kirschhaine winden, Brücken überqueren, zu Teehäusern und durch Laubengänge führen und dabei immer wieder Blicke auf den Teich gewähren (Abb. 10, Chesshire 2007). Sehr beliebt waren auch Trittsteine, auf denen man den Teich durchqueren konnte, sowie kunstvoll angelegte kleine Wasserfälle und Bäche. Neue wichtige Stilelemente der Wandelgärten waren Laternen, Wasserbecken und Teehäuser (RoBinson 2004). Baumbewachsene Inseln wurden oftmals durch Brücken mit dem Ufer und untereinander verbunden (Abb. 11). Pflanzen, die man für die Gestaltung der Wandelgärten verwendet, sind Kirschbaum-Arten (z. B. Prunus subhirtella). Gehölze mit schöner

Abb. 10 (oben): Vom Wandelpfad entlang des Sees im Ginkaku-ji Garten eröffnen sich immer wieder wunderschöne Blicke auf das Wasser mit effektvoller Spiegelung.

Abb. 11 (Mitte): Chinesische Brücken verbinden in Wandelgärten oftmals angelegte Kieferninseln miteinander und mit dem Ufer, wie hier im Ginkaku-ji Garten in Kyoto.

Abb. 12 (unten): Bäume mit schöner Laubfärbung und auffälligen Früchten wie die Kakipflaume (Diospyros kaki) kommen am Seeufer sehr gut zur Geltung wie hier im Teichgarten von Ryoan-ji in Kyoto.
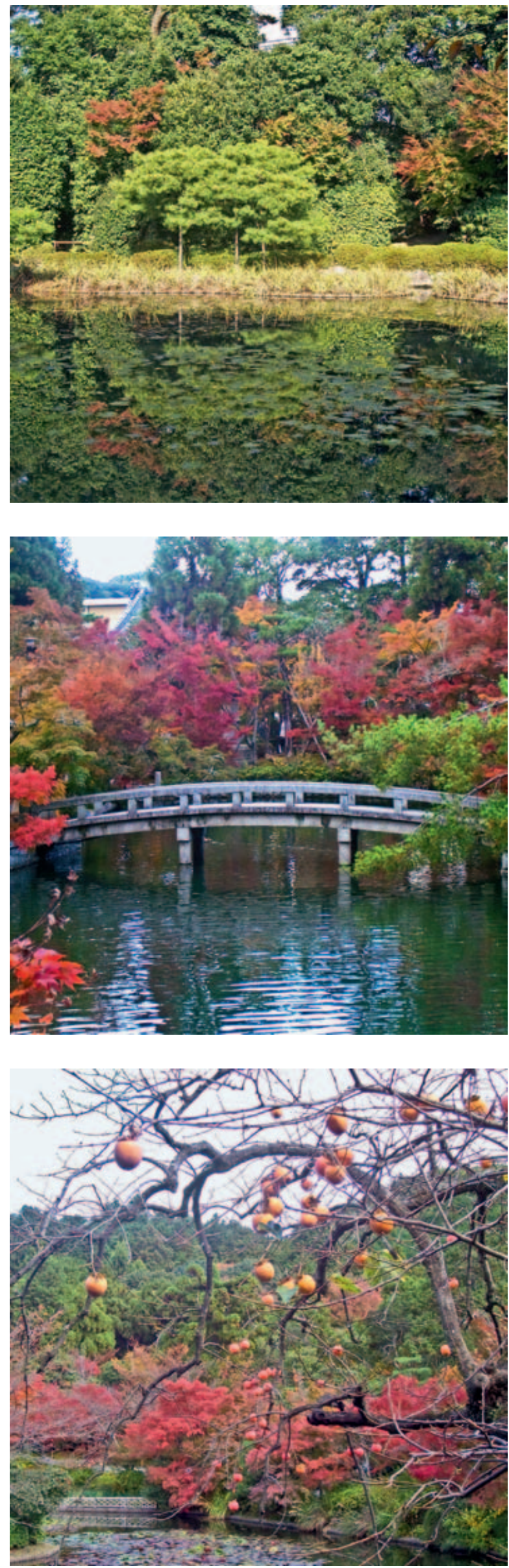


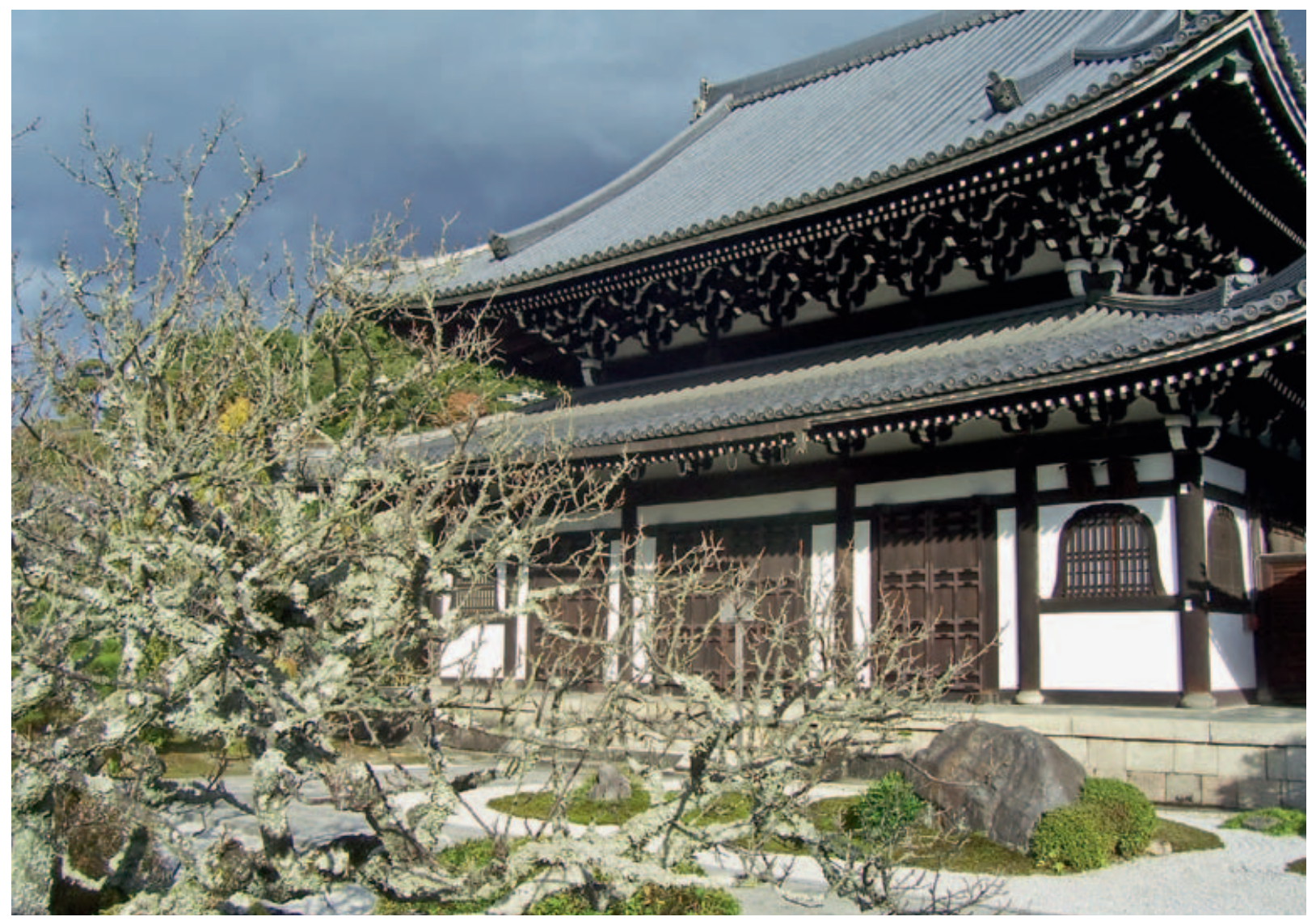

Laubfärbung für die Umsäumung von Wandelpfaden und Teichrändern sind z. B. Acer palmatum, Diospyros kaki, Nandina domestica oder Cercidiphyllum japonicum (Abb. 12). Glyzinien (Wisteria sinensis) dienen als Kletterpflanzen für Lauben und verschiedene Bambus-Arten werden zur Anlage kleiner Dickichte genutzt. Stauden und Geophyten finden sich im Uferbereich des Teiches und an Bachläufen. Typische Gewächse sind Iris-Arten (z. B. I. ensata), Lilien (z. B. Lilium auratum), Lotosblume (Nelumbo nucifera), Hortensien (Hydrangea macrophylla) und Funkien (Hosta spp.; Robinson 2004, Chesshire 2007).

Der bedeutendste Beitrag der MuromachiZeit zur Gartenkultur waren die Steingärten (Kare-sansui), welche unter dem Einfluss des Zen-Buddhismus entstanden und sich an alten japanischen Tuschezeichnungen orientierten (Keane 1999, Young \& Young 2006, Chesshire 2007). Bereits während der Kamakura-Zeit (1192-1333) existierten verschiedene Ausprägungen des Buddhismus, darunter die bekanntesten Hauptrichtungen Zen, Nichiren,
Jodo und Shingon. Zen gelangte im 13. Jh. durch Mönche von China nach Japan. Die ZenSekte sprach insbesondere den Stand der Samurai an. Die Mönche waren auf der Suche nach einer reineren Lehre ohne esoterische Praktiken wie etwa die Anbetung des Buddhas Amida. Der Zen-Buddhismus rückt mehr das Individuum und seine Bemühungen, den Geist insbesondere durch Meditation und die Erfahrung der "Nichtheit“ zu beherrschen (CHEsshire 2007) in den Mittelpunkt. Im späten 16. Jh. waren die japanischen Zen-Meister zu großen Garten-Künstlern avanciert. Sie waren durch chinesische und japanische Darstellungen von Trockengärten aus Sand und Felsen inspiriert. Ihre Schöpfungen wurden immer abstrakter und enthielten versteckte Botschaften des ZenBuddhismus.

Abb. 13: Der Eikando-Tempel in Kyoto wurde im 9. Jh. von der buddhistischen Shingon-Sekte errichtet. Er ist bekannt für seinen Amida-Buddha und den angrenzenden Steingarten. 


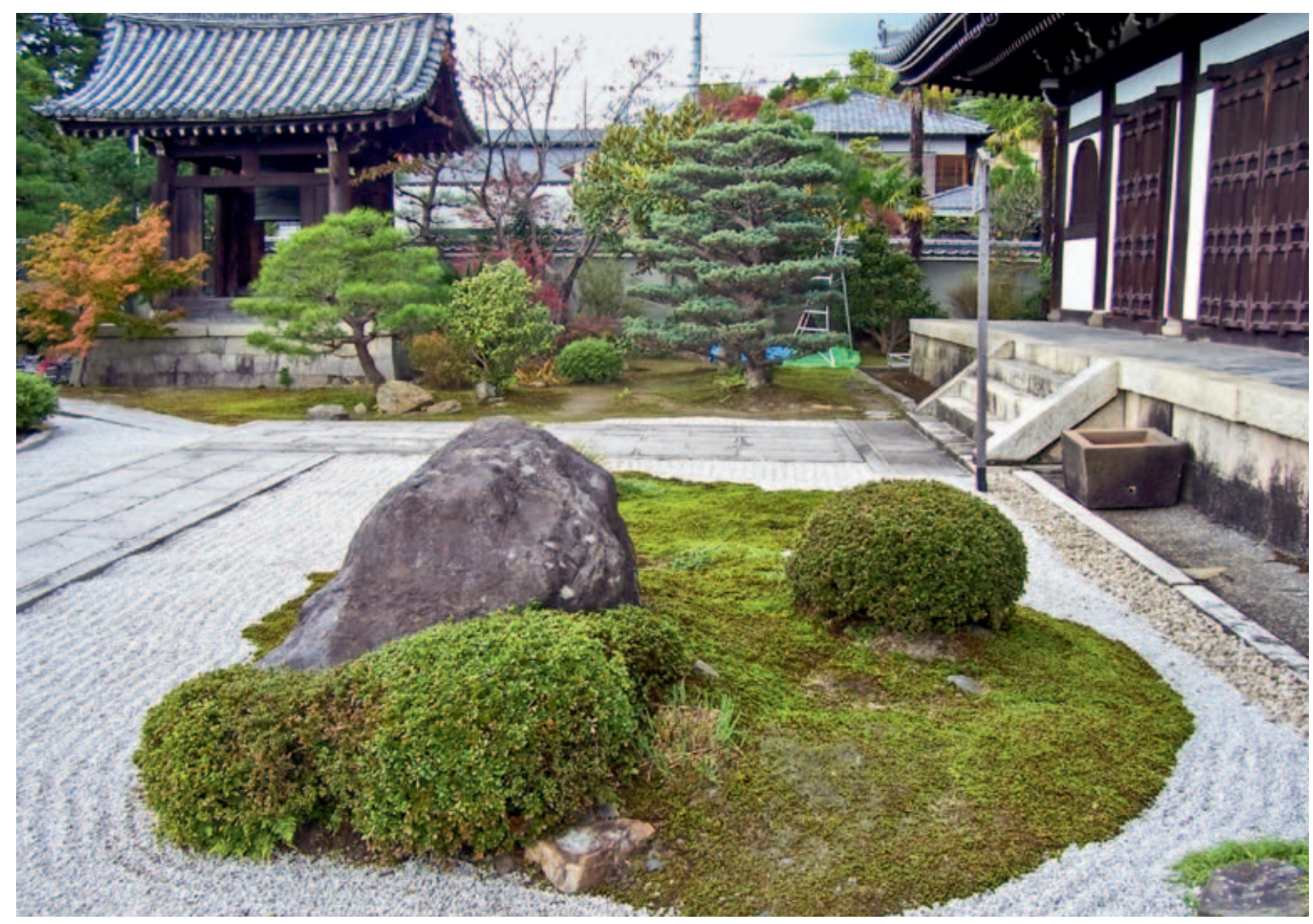

Der Ausgangspunkt für die Gestaltung eines Zen-Gartens ist immer der Tempel oder das Haus. Von einer bedachten Veranda aus, die von seitlichen Pfosten umrahmt ist, blickt man auf den darunter angelegten Garten wie auf ein Gemälde. Der von einem rechteckigen Rahmen eingefasste Garten mit Felsanordnung, Sand, Kies und Moosen ist eine künstlerische Komposition, die zur Meditation anregen soll (Abb. 13, 14, 15). Durch Meditation wird hier das Erleben von "Leere“ möglich, eines körperlosen Zustands des „Nicht-Ichs“, den Zen als das eigentliche Gesicht des Menschen definiert. Im Zen-Garten sehen Meditierende eine Widerspiegelung des unendlichen Raums (Keane 1999, Chesshire 2007).

Ein wichtiger Gestaltungsaspekt des Steingartens ist die Einbeziehung der umgebenden Landschaft. Gibt es beispielsweise eine Fernsicht auf den heiligen Berg Fujisan oder einen

Abb. 14: Im Steingarten des Eikando-Tempels wird das Meer durch den geharkten Kieskreis angedeutet. Ein heiliger Berg ragt auf einer mystischen Insel empor. anderen Berg, so wird dieser integriert und „mitgefangen“. Das Prinzip wird „shakkei“ („geborgte Landschaft") genannt (Young \& Young 2006, Chesshire 2007).

Die berühmtesten Zen-Gärten in Kyoto sind Daisen-in (um 1513 entstanden) und Ryoan-ji (1499 entstanden). Der rechteckige Steingarten Ryoan-ji wird von einer ölgetränkten LehmMauer umgrenzt. Über den gesamten inneren Raum ist feiner, silbrig grauer Quarzsand verteilt. Er wird täglich der Länge nach geharkt, so dass parallele Linien entstehen. In diesem Sandmeer sind 15 Steine angeordnet, die zu 5 moosgesäumten Gruppen in der Anordnung 5-2-32-3 zusammengefasst sind (Abb. 16). Diese Zahlenkombination hat im Buddhismus eine besondere Bedeutung, sie findet sich auch in Gesängen und Musikrhythmen wieder. Über die magische Anordnung der Steine gibt es viele verschiedene Theorien, aber bis heute kennt noch niemand ihre genaue Bedeutung. Steingärten bestehen nicht ausschließlich aus Steinen, Sand und Kies, sondern enthalten durchaus auch Pflanzen. Neben Moosen werden z.B. 

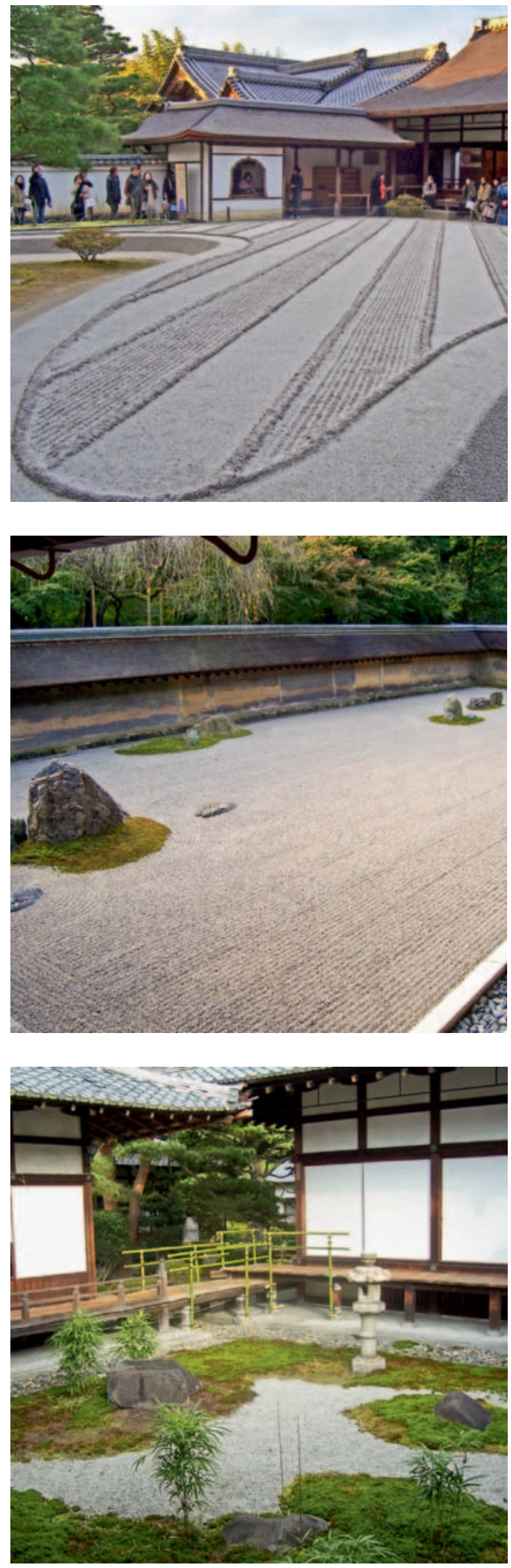

häufig polsterförmig wachsende Pflanzen verwendet, beispielsweise geschnittene Azaleen und für den Hintergrund eines Steingartens auch kleinwüchsige Kiefern, Bambus-Arten oder Kirschbäume (CHEsshire 2007).

Aufgrund ihres Minimalismus sind Steingärten heutzutage sehr modern und insbesondere in Städten sehr beliebt, wo oft nur kleine Flächen für die Gartengestaltung zur Verfügung stehen. Diesbezüglich nennenswert ist auch der kleinste aller japanischen Gärten, der so genannte Hofgarten, der ebenfalls sehr einfach gehalten ist und leicht zu gestalten ist: ein bis zwei Felsen, eine Laterne, ein Bambus oder eine Kiefer - mehr ist für die schlichte Schönheit eines solchen Gartens nicht notwendig (Abb. 17, Stahl 2006, Chesshire 2007).

\section{Literatur}

Chesshire, C. 2007: Japanische Gärten gestalten. -

München.

Giesen, W., Hassenpflug, W., Khan, K., Nahm, P. M., SChneider, W. \& Zollickhofer, J. 2007: Japan. Ostfildern.

Keane, M. P. 1999: Gestaltung japanischer Gärten. Stuttgart.

Robinson, P. 2004: Encyclopedia of water gardening. London.

STAHL, H. 2006: Bonsai. - Stuttgart.

Young, D. \& Young, M. 2006: Die Gärten Japans.

Tradition und Moderne. - Stuttgart.

\section{Internetseiten}

http://kosugi-zohen.co.jp/; darin: Büro für Landschaftsarchitektur und Landschaftsgestaltung, Kosugi-Zohen in Tokio

http://learn.bowdoin.edu/japanesegardens/; darin: Zur Geschichte verschiedener japanischer Gärten.

http://www.geocities.com/roman.jost/Japan_Allgemein/ Ryoanji.htm; darin: Zum Steingarten von Ryoan-ji: http://www.jgarden.org/; darin: Englische Website über Japanische Gärten - mit Glossar und vielen eingetragenen Gärten.

Abb. 15 (oben): Ein stilistisch etwas abgewandelter Steingarten des Kodai-ji-Tempels in Kyoto.

Abb. 16 (Mitte): Der Ende des 15. Jh. angelegte, weltbekannte Steingarten Ryoan-ji in Kyoto.

Abb. 17 (unten): Ein einem Kloster in Kyoto zugehöriger japanischer Hofgarten, der trotz kleiner Fläche durch die Schlichtheit seiner Gestaltung eine sehr effektvolle Wirkung hat. 